\title{
ENDOSCOPIC CORONARY ARTERY BYPASS GRAFTING WITH THE AID OF ROBOTIC ASSISTED INSTRUMENTS
}

Didier Loulmet, MD

Alain Carpentier, MD, $\mathrm{PhD}$

Nicola d'Attellis, MD

Alain Berrebi, MD

Cyril Cardon, MD

Olivier Ponzio, MD

Bertrand Aupècle, MD

John Y. M. Relland, MD
Objective: The development of endoscopic coronary artery bypass grafting has been limited because of poor visualization and increased technical difficulties in carrying out operations through ports. We investigated whether the use of robotic assisted instruments could minimize these difficulties. Methods: After a period of technical development and training on cadavers $(n=8)$ with the Intuitive Surgical system (Intuitive Surgical, Inc, Mountain View, Calif), the first clinical application in coronary artery surgery was performed in 4 male patients (mean age 59 \pm 6 years) with the indication of grafting the left internal thoracic artery to the left anterior descending coronary artery. Robotic assisted 3dimensional endoscopes and instruments were introduced into the left side of the chest through 3 intercostal ports. The Heartport system (Heartport, Inc, Redwood City, Calif) was used for arresting the heart during the anastomosis. Results: In 2 patients, the harvesting of the left internal thoracic artery was completed endoscopically with robotic assisted instruments and the anastomosis to the left anterior descending artery was performed through a minithoracotomy with conventional instruments. In 2 other patients, the entire operation was completed endoscopically with robotic assisted instruments. Early postoperative coronary angiography demonstrated the patency of the grafts in all cases. At 6-month follow-up, all patients were free of symptoms. Conclusions: Robotic assisted instruments make endoscopic coronary bypass possible and open a new era in minimally invasive surgery. ( $\mathrm{J}$ Thorac Cardiovasc Surg 1999;118:4-10)
C oronary artery bypass grafting $(\mathrm{CABG})$ has been associated with excellent long-term results, particularly when the left internal thoracic artery (LITA) has been used. ${ }^{1}$ Angioplasty, an alternative to surgical treatment, carries a higher incidence of failure because of recurrent obstruction of the dilated segment. ${ }^{2}$ However, this method is favored by the patients because of its noninvasive characteristics. Less invasive coronary artery surgery techniques developed in the past few years aim at combining the advantages of CABG and interventional cardiology techniques. Minimizing the

From the Department of Cardiovascular Surgery and Organ Transplantation, Hôpital Broussais, Paris, France.

Received for publication Jan 5, 1999; revisions requested Feb 4, 1999; revisions received March 5, 1999; accepted for publication March 10, 1999.

Address for reprints: Didier Loulmet, MD, Department of Cardiovascular Surgery and Organ Transplantation, Hôpital Broussais, 96, rue Didot_-75014 Paris, France.

Copyright (C) 1999 by Mosby, Inc.

$0022-5223 / 99 \$ 8.00+0 \quad \mathbf{1 2 / 1 / 9 8 4 2 2}$ incision and the deleterious effects of cardiopulmonary bypass (CPB) are the two main elements of this new strategy. The ultimate goal is to perform CABG endoscopically without CPB. An intermediary step is the completion of $\mathrm{CABG}$ through three $1-\mathrm{cm}$ ports with the heart arrested during the anastomosis. Attempts with this technique were confronted with problems of limited visualization and increased technical difficulties when using standard endosurgery instruments with limited range of motion. ${ }^{3}$ We investigated whether robotic assisted endoscopes and instruments could minimize these difficulties. After extensive evaluation on cadavers with the Intuitive Surgical system (Intuitive Surgical, Inc, Mountain View, Calif), the first endoscopic $\mathrm{CABG}$ in the human being was performed in June 1998. The experimental evaluation and early clinical experience form the basis of this report.

\section{Experimental study}

Material and methods. The Intuitive Surgical system has two original features: The surgeon is seated at a console look- 


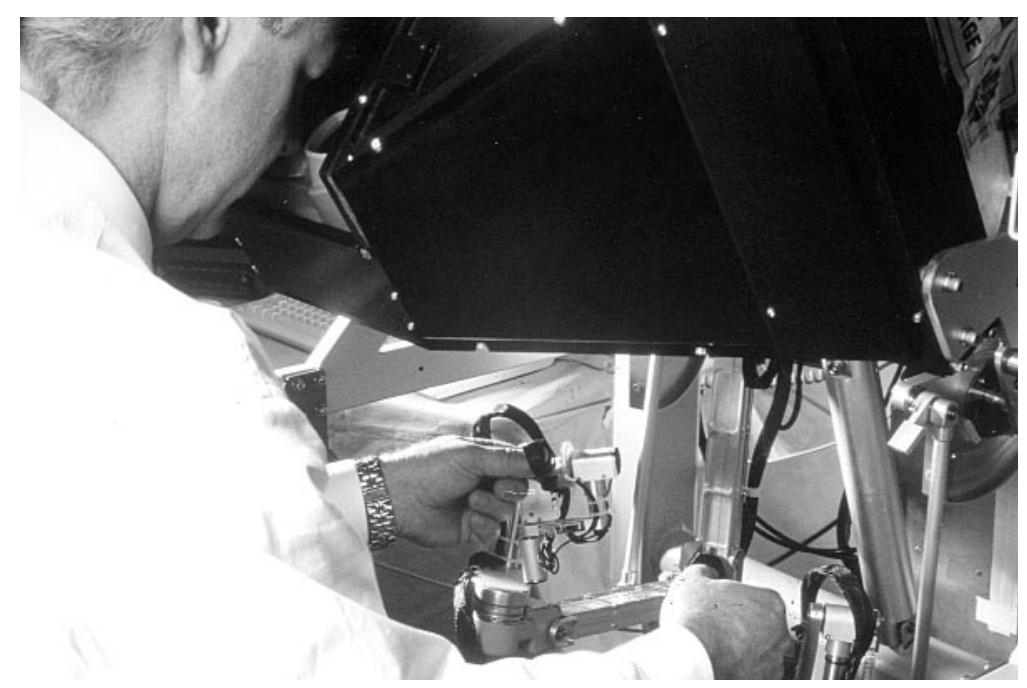

Fig 1. Surgeon operating while looking at the 3-dimensional binocular display of the operative field and manipulating instrument controllers.

ing at a 3-dimensional binocular display of the operative field and manipulating instrument controllers positioned under the display (Fig 1); the telemanipulated instruments supported by mechanical arms are articulated at their distal extremity so as to reproduce the motion of the surgeon's hands (Fig 2). A table side cart supports the three interactive mechanical arms. One arm is used to position a 3-dimensional endoscope and the two others are used to hold the removable articulated instruments. The visualization system is made up of two 3-chip cameras mounted on a custom 3-dimensional endoscope with two separate optical channels. Independently acquired images are transmitted to a high-resolution binocular display of the operative field. The operative image magnification may be up to 10 times the actual size. In terms of motion, the mechanical wrist of the instruments has 7 degrees of freedom. Tip articulations mimic the up/down ("pitch") and the side-to-side ("yaw") flexibility of the human wrist. Instrument tips in the display are electronically aligned with the instrument controllers to ensure the hand-eye orientation and natural operative feeling found in open surgery. System electronics allow the use of motion scaling: the surgeon's hand movements are transmitted to the corresponding instruments with a $3: 1$ or $5: 1$ reduction ratio. The computer system eliminates occasional tremor.

The purpose of this experimental phase on 8 cadavers was to solve the most difficult challenge of totally endoscopic coronary surgery - the proper placement of the ports. Ports were located so as to perform both the LITA harvesting and the LITA-LAD anastomosis with robotic assisted instruments (RAI) through 3 ports without the help of an assistant. On each cadaver, an average of 7 ports were used to determine the optimal combination of 3 ports.

\section{Results}

Port placement. The first port (camera) had to be placed so that the endoscope faced the anastomotic site so as to visual- ize the entire length of the LITA to be dissected. We found that these conditions were achieved by placing the port in the fourth intercostal space at the level of the midclavicular line. The second port (right instrument) had to be positioned so that the right instrument reached the middle third of the LAD and both extremities of the LITA, staying on the right of the axis of the endoscope. These conditions were satisfied when the second port was positioned through the fourth $(n=7)$ or the third $(n=1)$ intercostal space at the anterior axillary line. The third port (left instrument) had to be placed so that the left instrument reached the middle third of the LAD and both extremities of the LITA, staying to the left of the axis of the endoscope. These conditions were satisfied when the third port was positioned through the sixth $(n=6)$ or the seventh $(\mathrm{n}=2)$ intercostal space at the anterior axillary line.

LITA dissection and preparation. In all cases $(n=8)$, exposure and access to the proximal portion of the LITA was simple. However, in 6 cases, dissection of the distal portion of the LITA was difficult because of limited space between the anterior part of the pericardium and the chest wall owing to a narrow anatomic angle $(n=4)$ or to cardiomegaly $(n=2)$. Three techniques were tried to improve the exposure of the distal portion of the LITA: (1) Dissecting the anterior attachment of the pericardium to the sternum had the inconvenience of opening the right pleural cavity; (2) the use of a fan-shaped retractor to push the pericardium away from the chest wall caused interference with the left instrument and had the inconvenience of adding an additional port in the subcostal location; (3) carbon dioxide insufflation was the only technique that significantly improved the exposure of the distal segment of the LITA by increasing the space between the anterior pericardium and the chest wall. On the basis of these results, the ports of the instruments were modified to allow carbon dioxide insufflation and airtightness. 


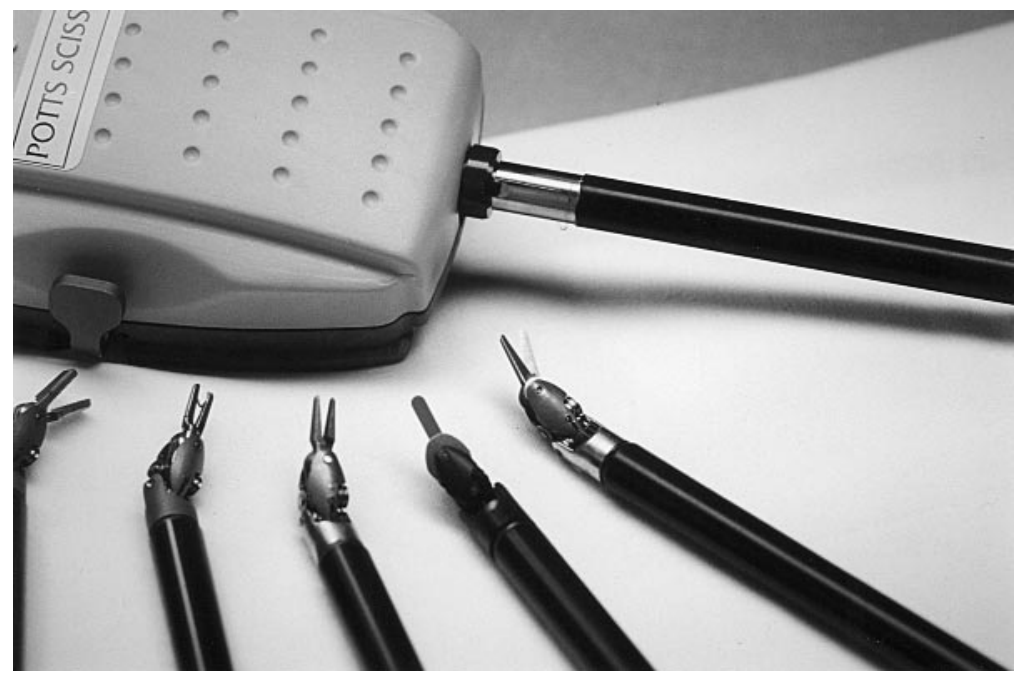

Fig 2. Set of instruments showing the "endo-wrist" or distal articulation.

After extensive dissection of the LITA, it was found useful to exteriorize the distal extremity outside the thorax through the most proximal port to prepare it for the anastomosis. Inspection of the LITA pedicle showed a satisfactory result in all the cases except one in which a tear of a collateral branch resulted from a technical error in the placement of a clip. The jaws of the clip-applier were redesigned so as to facilitate the placement of the clips.

Arteriotomy and anastomosis. After the pericardium had been opened longitudinally, the identification of the LAD was difficult at the beginning of the experimental phase. The distance between the endoscope and the heart was insufficient to provide an overall view of the left ventricle including the LAD and its branches. Moreover, the diagonal branches could be mistaken for the LAD because of magnification. To facilitate LAD identification, the LAD had to be followed with the endoscope from its origin between the pulmonary artery and the left atrial appendage, up to the apex of the heart. Once the coronary artery had been identified and incised, the completion of the anastomosis was not difficult. The only drawback was the absence of tactile feedback, which made it difficult to apply appropriate tension on the two ends of the suture when tying the knot. There was a risk of purse-string effect at the site of the anastomosis or suture break by applying excessive tension. This inconvenience was minimized by training, replacing the tactile feeling by visual control when exerting traction on the two ends of the suture.

\section{Clinical study}

Patients and methods. From June 26, 1998, to July 2, 1998, 6 patients with the surgical indication of a single CABG to the LAD were given the choice between the standard and the endoscopic techniques using RAI. Two patients chose the standard technique and 4 patients accepted operations with the endoscopic technique and duly signed the con- sent form approved by the Ethics Committee (ref. 1443-3-31998, Direction des Hôpitaux de Paris). These 4 patients were men with an average age of $59 \pm 6$ years. They had a significant stenosis of the LAD, associated in 2 cases with an occlusion of the right coronary artery. In these 2 cases, revascularization of the posterior vessels was not deemed necessary because of their small size and of a previous posterior myocardial infarction. They were scheduled for complete endoscopic LITA-LAD grafting with angiographic examination before discharge. Follow-up was by their referring cardiologists after discharge. Charts, operative reports, and intraoperative video recordings of the 4 patients were retrospectively reviewed.

Anesthesia. Induction of anesthesia was performed with a target controlled infusion of remifentanil (target plasma concentration $10 \mathrm{ng} / \mathrm{mL}$ over 2 minutes) and propofol (target plasma concentration $1 \mu \mathrm{g} / \mathrm{mL}$ over 3 minutes). Intubation was performed with a 37F left-sided Robert Shaw doublelumen endotracheal tube so as to exclude the left lung during LITA dissection. After intubation, a 9F introducer was placed into the right internal jugular vein through which an $8.3 \mathrm{~F}$ Endopulmonary Vent catheter (Heartport) was positioned in the main pulmonary artery. A multiple plane transesophageal echocardiography probe was placed after all central lines had been inserted. Anesthesia was maintained with a target-controlled infusion of remifentanil $(7 \mathrm{ng} / \mathrm{mL})$ and propofol (1 $\mu \mathrm{g} / \mathrm{mL}$ ). Postoperative sedation consisted of propofol (3 $\mathrm{mg} / \mathrm{kg}$ per hour), allowing patients to enter a fast-track recovery and early extubation protocol.

Surgical technique. The patient was placed in the supine position with the left arm extended above the head. External defibrillation pads were placed on the chest wall. The right femoral vessels were exposed. After the left lung had been collapsed, the first port (camera) in the left fourth intercostal space at the midclavicular line was insufflated with carbon 


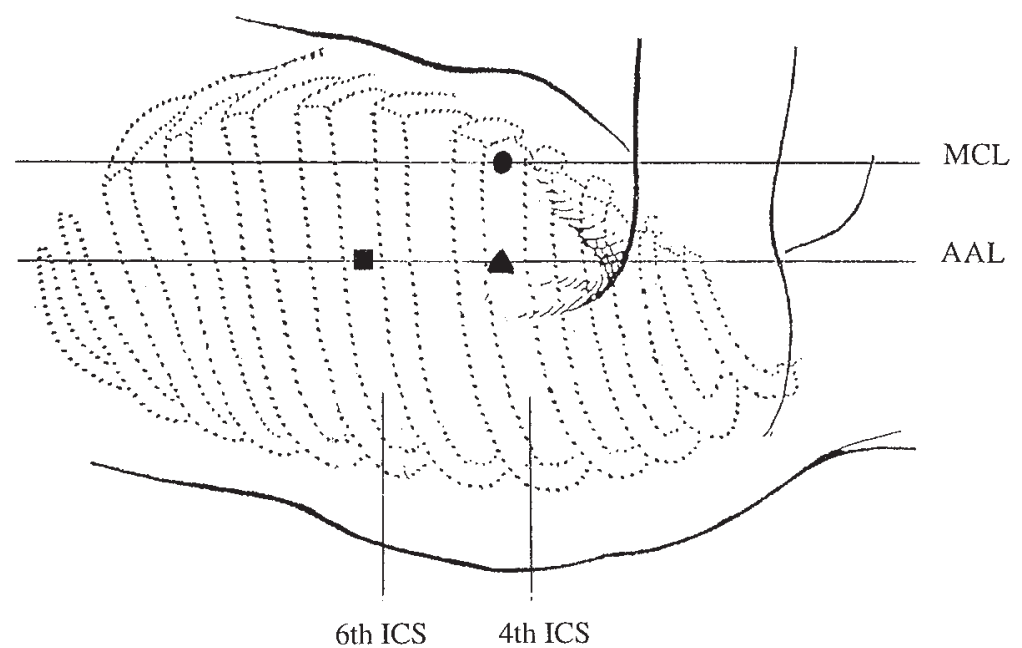

Fig 3. The position of the 3 ports used for the operation. $A A L$, Anterior axillary line; $M C L$, midclavicular line; ICS, intercostal space; circle, camera port; diamond, right instrument port; square, left instrument port.

dioxide until a 5-mm $\mathrm{Hg}$ intrapleural pressure was obtained. The left pleural cavity was explored with an endoscope. The second port (right instrument) was placed in the fourth intercostal space at the level of the anterior axillary line. The third port (left instrument) was inserted in the sixth intercostal space at the level of the anterior axillary line (Fig 3).

The surgical arms of the Intuitive Surgical system were placed in the thorax and the LITA dissection was started with RAI (3-dimensional 30-degree endoscope, electrocautery, and grasper) with a 3:1 motion reduction ratio. Carbon dioxide insufflation was continued at a pressure of $5 \mathrm{~mm} \mathrm{Hg}$. The LITA was dissected from the first costal cartilage to the fifth intercostal space. The collateral branches of the thoracic artery were divided mainly by means of electrocautery. After heparinization, the distal end of the LITA was divided between clips. The instruments and the mechanical arms were then removed from the thorax. An Endobulldog clamp (Heartport) was placed on the proximal segment of the LITA through the upper port. Preparation of the distal end of the LITA was by conventional open technique after exteriorization of the distal tip through the upper port.

The arterial and venous cannulas (Heartport) were introduced into the right femoral vessels. The Endoaortic clamp (Heartport) was placed in the ascending aorta with the aid of transesophageal echocardiography. The surgical arms of the Intuitive Surgical system were replaced within the left side of the chest. After CPB was established $\left(34^{\circ} \mathrm{C}\right)$ and the ventilation stopped, the pericardium was opened with RAI (3dimensional 0-degree endoscope, electrocautery, and grasper). When the LAD was identified, the Endoaortic clamp was inflated and crystalloid cardioplegic solution was delivered. After cardiac arrest, the LAD was dissected and opened with the use of RAI (3-dimensional 0-degree endoscope, bevel, and sharp blades) with 5:1 motion reduction ratio. The anastomosis was completed with a $65-\mathrm{mm} 7.0$
Coronyl suture (Laboratoires Peters, Bobigny, France) with RAI (small forceps and needle holder) with a 5:1 motion reduction ratio. After the Endobulldog clamp had been released and the anastomosis checked for bleeding, the Endoaortic clamp was deflated, double lung ventilation was resumed, and the patient was weaned from $\mathrm{CPB}$. The first and the second port orifices were closed. A chest tube was placed in the third port.

\section{Results}

Two patients underwent a complete endoscopic operation with RAI, including the dissection the LITA and completion of the anastomosis to the LAD on the arrested heart. In the 2 other patients, the LITA was dissected endoscopically with RAI and the anastomosis was made through a minithoracotomy $(5 \mathrm{~cm})$ in the fourth intercostal space by means of conventional techniques. In the latter 2 cases, conversion for the second stage of the operation was necessary because of the difficulty in localizing an intramyocardial LAD in 1 case and the impossibility of introducing the guide wire of the Heartport Endoaortic clamp in either iliac vessel in the other case. In these 2 cases, the anastomosis was completed under CPB with the heart arrested in the first case and decompressed in the second.

In the 4 patients, the time necessary for harvesting endoscopically the LITA averaged $78 \pm 13$ minutes. This included the time for setting up the system and dissecting the LITA. Several times during the dissection, the motion of the mechanical arms was limited by two types of interference: (1) interference between the mechanical arms and (2) interference between the mechanical arms 


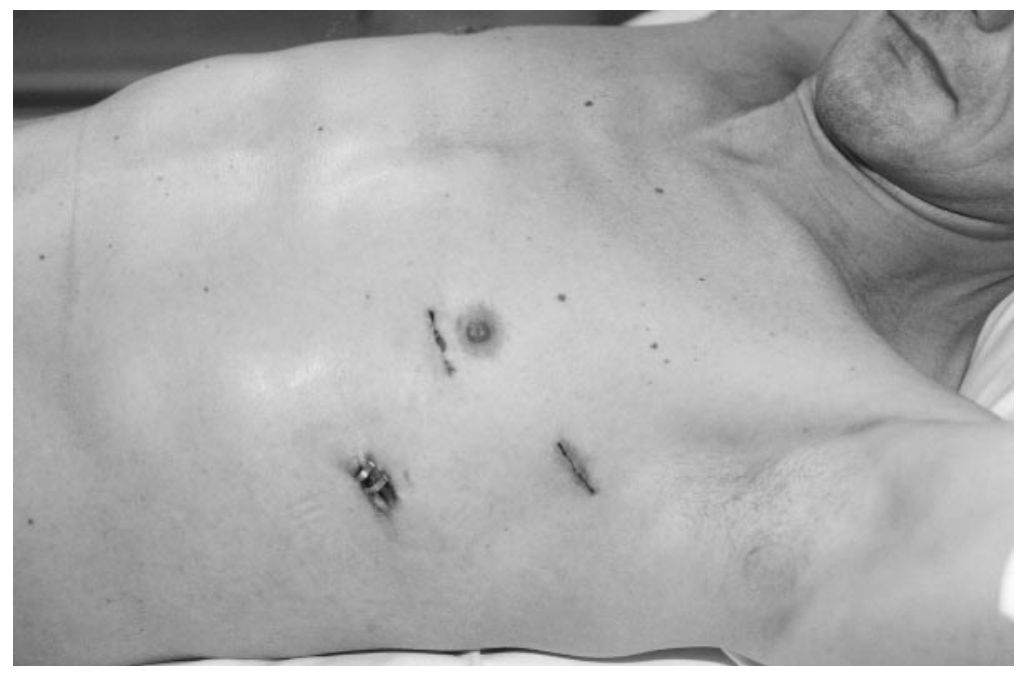

Fig 4. Chest of the patient 3 days after complete endoscopic surgery.

and the body of the patient. Each time, this obliged us to manually reposition the arms of the system.

The preparation of the distal extremity of the LITA took an average of $25 \pm 8$ minutes. It was difficult to take the distal end of the pedicle out of the chest without twisting it. The exteriorized portion of the LITA was short and it was crucial to have no internal twist to be able to make the correct distal incision on the appropriate side of the artery.

In the 2 patients having the coronary anastomosis carried out endoscopically, the anastomosis took 18 minutes in the first and 32 minutes in the second. In the second patient, exposure was not optimal because the middle third of the LAD was intramyocardial, which meant performing the anastomosis distally in a region that was not optimal in relation to the endoscope and the instruments. Otherwise, suturing did not present any difficulty per se.

Cardiac arrest lasted 47 and 65 minutes. CPB time was 80 and 100 minutes. Time was lost in correctly positioning the LITA before anastomosis. In the absence of an assistant, the LITA pedicle had a tendency to slide off the pericardium into the thorax and to get twisted. It was difficult then to return it to proper position with RAI.

The 2 patients remained in the intensive care unit for 24 hours and were extubated at the sixth and ninth postoperative hours. Blood loss was $345 \mathrm{~mL}$ and 395 $\mathrm{mL}$, and no transfusion was required. The lengths of stay in the hospital were 7 days and 6 days. The first patient did not have any postoperative pain and refused pain medication. The second patient had a paresthesia with associated pain of the left arm resulting from the position during the operation. This disappeared completely after 3 days. Fig 4 shows the chest of this patient 3 days after the operation.

The 2 patients in whom conversion to a minithoracotomy was necessary had an uneventful postoperative course and were discharged on the seventh and the eighth postoperative days.

The 4 patients underwent angiographic examination before discharge, which demonstrated patency of the anastomoses in all cases. At 6 months' follow-up these patients were symptom free.

\section{Discussion}

The introduction of robotics in cardiac surgery was predicted 15 years ago. ${ }^{4}$ The development by Intuitive Surgical, Inc, of a robotic system incorporating not only robotic assisted visualization but also robotic assisted instrumentation prompted us to cooperate with this company. This cooperation led to the first robotic assisted intracardiac operation in human beings in May 1998. ${ }^{5}$ The first application of this technology to coronary artery surgery to our knowledge took place in June 1998 and is the subject of this report.

Current surgical techniques can be separated into open and endoscopic techniques. In open surgery, the operator uses his natural 3-dimensional vision and the 7 degrees of freedom of his hand movements. In endoscopic surgery, looking at a monitor and using conventional endoinstruments, the operator loses his visual perception of depth and the natural hand-eye coordination. In terms of motion, the classic endoinstruments have only 5 
degrees of freedom. Moreover, the hand of the surgeon and the tip of the instruments move in opposite directions. These limitations have restricted the use of endosurgery techniques to mainly excisional procedures.

The Intuitive Surgical system uses "master-slave robotics." Its kinematic (or joint movements) structure allows the surgeon to use his traditional open surgery techniques at the console ("master"), which are simultaneously reproduced using endosurgery movements by the instruments (slaves) at the surgical site with 7 degrees of freedom. In other words, the system acts as a translator of open surgery techniques into endosurgery techniques. Other systems of robotic assisted visualization and instrumentation have specific features that differ significantly from those of the Intuitive Surgical system. The Aesop system (Computer Motion, Goleta, Calif) provides voice-driven positioning of the thoracoscope, particularly useful in less invasive thoracic surgery. The Zeus system (Computer Motion) provides RAI but with limited motion at the extremity of the instruments, which are not articulated. Up to now, no completely endoscopic CABG has been reported using these systems. ${ }^{6}$ In this experience, the entire operation including LITA dissection and LAD anastomosis was possible through 3 ports with the use of RAI in 2 patients with good results. Several points merit further discussion, however.

LITA harvesting. Compared with the various minimally invasive techniques used for LITA harvesting, several features of the Intuitive Surgical system were found useful in increasing the precision of this procedure. ${ }^{7-9}$ High magnification allowed dissection of a very thin vascular pedicle without injuring the intercostal muscles or the periosteum of the costal cartilages. Three-dimensional vision provided a much better perception than 2-dimensional visualization as far as spatial orientation of the vessels and the instruments were concerned. The "mechanical wrist" allowed for a full range of motion of the tip of the instruments, which facilitated the dissection in remote areas such as the proximal and distal extremities of the LITA pedicle.

Yet, interference between the arms of the system or between the arms and body of the patient during the dissection were frequent. In the future, miniaturization of the architecture of the arms should solve this problem. In addition, preparation of the distal extremity of the LITA after exteriorization was a source of lost time because of twisting of the pedicle. An endoscopic technique for this part of the procedure should minimize the risk of twisting.

Target vessel exposure. Different techniques have been developed to immobilize the site of the anastomo- sis and to occlude the target vessel while operating on a beating heart. ${ }^{10-14}$ These techniques imply the use of a thoracotomy or a sternotomy. Because the aim of this operation was primarily to use closed chest technique, the Heartport system was used to immobilize the heart and to obtain a dry operative field. ${ }^{15}$

In this preliminary experience, the interval between LITA dissection and anastomosis was particularly long because of the time required to set up the Heartport system and to localize the target vessel. This can be reduced in the future by setting up the Heartport system while dissecting the LITA and by using cameras with wider-angle zooming capability, giving an overall view of the left ventricle with the usual anatomic landmarks to find the LAD.

Completion of the anastomosis. The capability of the system was fully appreciated during the completion of the anastomosis. The distal articulation of the instruments allowed perpendicular suture needle positioning to the arterial tissue in all cases. Image magnification allowed observation of important details that cannot be seen in open surgery, such as microclots or microdroplets of fat present in the anastomotic site. Threedimensional vision also allowed perfect control of the needle trajectory during suturing.

In the absence of an assistant, correctly positioning the LITA at the beginning of the anastomosis was a problem. To avoid sliding and twisting of the pedicle, we recommend suturing the LITA pedicle to the epicardium before the anastomosis.

\section{Conclusion}

This preliminary experience shows that robotic assisted visualization and instrumentation can minimize some of the difficulties and limitations associated with totally endoscopic CABG. Precision of surgical technique is a significant advantage. Several problems became evident and should be addressed in the future: cumbersome devices, interferences between the arms of the system, heavy technical assistance, and poor tactile feeling. Reducing these drawbacks should expand the use of and indications for totally endoscopic CABG.

\section{REFERENCES}

1. Yusuf S, Zucker D, Peduzzi P, Lloyd DF, Takaro T, Kennedy JW. Effect of coronary artery bypass graft surgery on survival: overview of 10-years results from randomized trials by the coronary artery bypass graft surgery trialists collaboration. Lancet 1994; 344:563-70.

2. Pocock SJ, Henderson RA, Richards AF, Hampton JR, King SB, Hamm CW. Meta-analysis of randomized trials comparing coronary angioplasty with bypass surgery. Lancet 1995;346:1184-9.

3. Reitz BA, Stevens JH, Burdon TA, St Goar FG, Siegel LC, Pompili 
MF. Port-access coronary artery bypass grafting: lessons learned in a phase I clinical trial [abstract]. Circulation 1996;94(Suppl):I52.

4. Carpentier A. Cardiac valve surgery - the "French correction." J Thorac Cardiovasc Surg 1983;86:323-37.

5. Carpentier A, Loulmet D, Aupècle B, Kieffer JP, Tournay D, Guibourt $\mathrm{P}$, et al. Chirurgie à coeur ouvert assistée par ordinateur. Premier cas opéré ave succés. CR Acad Sci Paris, Sciences de la vie/Life Sciences 1998;321:437-42.

6. Stephenson ER, Sankholkar S, Ducko CT, Damiano RJ. Robotically assisted microsurgery for endoscopic coronary artery bypass grafting. Ann Thorac Surg 1998;66:1064-7.

7. Calafiore AM, DiGiammarco G, Theodori G, Bosco G, D'Annunzio E, Barsotti A, et al. Left anterior descending coronary artery grafting via left anterior small thoracotomy without cardiopulmonary bypass. Ann Thorac Surg 1996;61:1658-65.

8. Benetti FJ, Ballester C, Sani G, Boonstra PW, Grandjean JG. Videoassisted coronary bypass surgery. J Cardiol Surg 1995;10:620-5.

9. Nataf P, Lima L, Regan M, Benarim S, Ramadan R, Pavie A, et al. Thoracoscopic internal mammary artery harvesting: technical consideration. Ann Thorac Surg 1997;63:S104-6.
10. Borst C, Jansen EWL, Gründeman PF, Van Dogen JWF, Masvel Beck HJ, Wesenhagen H. Regional cardiac wall immobilization for open and closed chest coronary artery bypass grafting on the beating heart: the "Octopus" method [abstract]. Circulation 1995; 92(Suppl):I177.

11. Abhay SW, Kole SD. Clamp for coronary artery operations. Ann Thorac Surg 1998;65:1475-6.

12. Rivetti LA, Gandra SMA. Initial experience using an intraluminal shunt during revascularization of the beating heart. Ann Thorac Surg 1997;63:1742-7.

13. Heijmen RH, Borst C, Van Dalen R, Gründeman PF, Verlan CWJ. Temporary luminal arteriotomy seal for bypass grafting. Ann Thorac Surg 1998;1093-9.

14. Mack MJ, Acuff TE, Casimir Ahn H, Lönn UJ, Jansen EWL. Video-assisted coronary bypass grafting on the beating heart. Ann Thorac Surg 1997;63:s100-3.

15. Stevens JH, Burdon TA, Peters WS, Siegel LC, Pompili MF, Vierra MA, et al. Port-access coronary artery bypass grafting: a proposed surgical method. J Thorac Cardiovasc Surg 1996;111: $567-73$. 